INPLASY

PROTOCOL

To cite: Guo et al. Effect of Dance-Based Mind-Motor Activities for post-stroke insomnia A protocol for systematic review and metaanalysis. Inplasy protocol

202210011. doi:

10.37766/inplasy2022.1.0011

Received: 02 January 2022

Published: 02 January 2022

Corresponding author:

Yi Ding

1253038007@qq.com

Author Affiliation:

The Second Affiliated Hospital

of Shandong University of

Traditional Chinese Medicine.

Support: Nothing.

Review Stage at time of this submission: Formal screening of search results against

eligibility criteria.

Conflicts of interest:

None declared.

\section{Effect of Dance-Based Mind-Motor Activities for post-stroke insomnia A protocol for systematic review and meta-analysis}

Guo, CC 1 ; Zheng, YK2; Wang, CA3 ; Ding, $Y^{4}$.

Review question / Objective: Post-stroke insomnia (PSI) is an important complication of stroke, which often affects patients in many aspects. Dance-based mind-motor activities were defined as coordinated upright mind-motor movements that emphasize dynamic balance, structured through music or an inner rhythm (eg, breathing) and distinctive instructions or choreography, and that involve social interaction. It has been gradually applied to the rehabilitation of post-stroke mental disorders and sleep disorders. The purpose of this study was to investigate the efficacy and safety of dance-based mindmotor activity in the treatment of PSI.

Condition being studied: PSI is a common complication of stroke, which often affects patients in various aspects. Therefore, it is necessary to intervene in PSI. In recent years, an increasing number of researchers have conducted clinical trials of dance-based mind-motor activities for PSI. Therefore, it is necessary to conduct a systematic review to establish convincing evidence to prove the effectiveness and safety of dance-based mind-motor activities for PSI.

INPLASY registration number: This protocol was registered with the International Platform of Registered Systematic Review and Meta-Analysis Protocols (INPLASY) on 02 January 2022 and was last updated on 02 January 2022 (registration number INPLASY202210011).

\section{INTRODUCTION}

Review question / Objective: Post-stroke insomnia (PSI) is an important complication of stroke, which often affects patients in many aspects. Dance-based mind-motor activities were defined as coordinated upright mind-motor movements that emphasize dynamic balance, structured through music or an inner rhythm (eg, breathing) and distinctive instructions or choreography, and that involve social 
interaction. It has been gradually applied to the rehabilitation of post-stroke mental disorders and sleep disorders. The purpose of this study was to investigate the efficacy and safety of dance-based mind-motor activity in the treatment of PSI.

Condition being studied: PSI is a common complication of stroke, which often affects patients in various aspects. Therefore, it is necessary to intervene in PSI. In recent years, an increasing number of researchers have conducted clinical trials of dancebased mind-motor activities for PSI. Therefore, it is necessary to conduct a systematic review to establish convincing evidence to prove the effectiveness and safety of dance-based mind-motor activities for PSI.

\section{METHODS}

Participant or population: The patients in the study will be those who are diagnosed with PSI at the age of 35 or older. There will be no restrictions on diagnostic criteria, race, sex, economic situation, and education level.

Intervention: Dance-based mind-motor activities, drug, other therapies.

Comparator: 1.The treatment group was given dance-based mind-motor activities, while the control group was given drug. 2 . The treatment group received dance-based mind-motor activities+ other therapies, while the control group received drug + other therapies.The symptomatic treatment of the treatment group was the same as that of the control group.

Study designs to be included: The included studies were randomized controlled design.

Eligibility criteria: The included studies were randomized controlled design.

Information sources: The electronic databases of Cochrane Library, MEDLINE, PubMed, Springer, EMBASE, Chinese Science Citation Database (CSCD), China National Knowledge Infrastructure (CNKI),
Chinese Biomedical Literature Database (CBM), Chinese Scientific Journal Data-b ase (VIP), Wan-fang database will be searched from the establishment to February 1, 2022.

Main outcome(s): Subjective sleep quality.

Quality assessment / Risk of bias analysis: To assess the risk of bias, we will use the Cochrane risk assessment tool.The following 7 items will be included: random sequence generation, allocation concealment, blinding of participants and caregivers, blinding of outcome evaluator, incomplete outcome data, selective reporting, and other bias. High, low, unclear assessments will be performed for each item.

Strategy of data synthesis: RevMan5.3.5 will be used for all statistical analyses. We will use the random effects model to merge the data. The results of the meta-analyses will be presented by forest plots. When the results are unsuitable to be combined due to the clinical or methodologic heterogeneity, we will perform descriptive analysis.

Subgroup analysis: In case of any significant heterogeneity among the preliminary result of data synthesis, consideration will be given to the subgroup analysis of age, stroke type, exercise style, exercise frequency, exercise duration, severity of insomnia, sample size and other factors.

Sensitivity analysis: When possible, we will conduct sensitivity analysis to ensure the stability and reliability of the conclusions drawn from the meta-analysis. Low quality experimental studies will be excluded, and if the synthetic results show high heterogeneity (the I2test is $>\mathbf{7 5 \%}$ ), it is necessary to consider re-evaluation to determine stability and reliability.If there is no significant change in the result of synthesis, we could consider that the synthesis are reliable.

Country(ies) involved: China. 
Keywords: dance-based mind-motor activities; PSI.

Contributions of each author:

Author 1 - Chenchen Guo.

Author 2 - Yuekun Zheng.

Author 3 - Congan Wang.

Author 4 - Yi Ding. 